\title{
Resonant Interactions between Shelf Waves, with Applications to the Oregon Shelf
}

\author{
William W. Hsieh and Lawrence A. Mysak ${ }^{1}$ \\ Department of Oceanography, University of British Columbia, Vancouver, British Columbia, Canada V6T IW5
}

(Manuscript received 1 April 1980, in final form 22 July 1980)

\begin{abstract}
From the inviscid, unforced, barotropic long-wave equations for a rotating system, it is shown that resonant interactions between three continental shelf waves can occur. Evolution equations governing the amplitude and the energy of individual waves in a resonant triad are derived. The nonlinearity in the governing equations allows energy to be transferred between the waves, but with the total energy conserved. While the shelf waves typically have periods of several days, the energy transfer has a time scale of order 12 days. Observational evidence of resonant shelf wave interactions on the Oregon shelf is found in the spectral analyses of Cutchin and Smith (1973) and Huyer et al. (1975), where their observed signals agree well with the resonant frequencies deduced from the theory. The good agreement between theory and observation suggests that nonlinear energy transfer may play a much more significant role in shelf wave dynamics than was previously realized.
\end{abstract}

\section{Introduction}

Low-frequency waves have often been observed propagating along continental shelves, with their energies trapped near the coast. Characterized by sub-inertial frequencies, these continental shelf waves, in the absence of strong mean flows, propagate their phase with the coast to their right in the Northern Hemisphere. Mysak (1980) gives a broad review of the recent theoretical and observational work in shelf wave dynamics.

Much of the research on the theory of shelf waves has been done using the long-wave equations in which the nonlinear terms have been omitted. However, during the past 15 years or so, many investigators studying other types of oceanic wave motion have found that the nonlinear terms in the governing equations can lead to wave-wave interactions where energy is transferred between different wave components. Discussions of this wave-wave interaction mechanism in the ocean are presented in Hasselmann (1968), Phillips (1977) and LeBlond and Mysak (1978, Section 38). In general, the energy exchanges due to the nonlinear terms are of order $\epsilon$, where $\epsilon$ is a (usually small) parameter arising from nondimensionalizing the governing equations (e.g., for shelf waves, $\epsilon$ is the Rossby number and is typically of order $10^{-2}$ ). The nonlinear energy exchanges, therefore, are usually small. However, when certain "resonance conditions" are satisfied by the waves, the energy exchanges can be much

\footnotetext{
${ }^{1}$ Also Department of Mathematics, University of British Columbia.
}

more appreciable. The lowest order resonant interaction involves three waves (a triad). It is well known that resonant triad interactions can occur between Rossby waves, internal waves and edge waves, but not between surface gravity waves (for which resonant interactions can occur only at higher order, i.e., between four or more waves). In this paper, we find that resonant interactions between three unforced shelf waves are possible (see also Mysak, 1980), and that there is observational evidence of such shelf wave interactions in the spectral analyses of Cutchin and Smith (1973) and Huyer et al. (1975).

One of the resonance conditions is that the angular frequencies $\omega_{1}, \omega_{2}$ and $\omega_{3}$ of the three shelf waves satisfy

$$
\omega_{1}+\omega_{2}=\omega_{3} .
$$

Cutchin and Smith (1973) observed three significant peaks at around $0.22,0.40$ and 0.65 cpd (cycles per day) in various cross spectra computed from Oregon coast data (Fig. 1). The resonance condition (1.1) is fairly well satisfied by these three observed frequencies. Buchwald and Adams (1968) noted that for shelf wave dispersion curves which have zero slope (i.e., zero group velocity) at certain frequencies, the wave energy cannot propagate away at these frequencies, and therefore, peaks in the frequency spectrum might be expected there. Cutchin and Smith (1973) attempted to apply the Buchwald and Adams explanation to their observed peaks, but concluded:

The restriction of shelf wave phenomena to only certain frequency bands remains as one of the most 


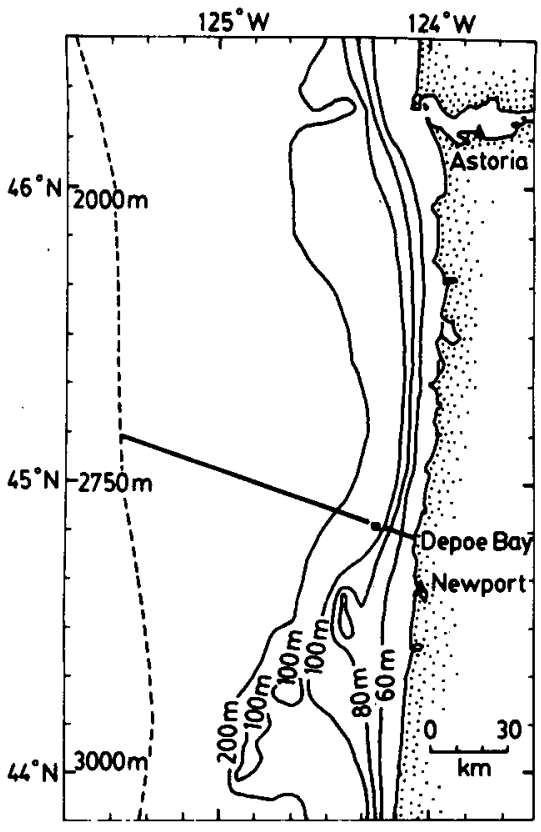

(a)

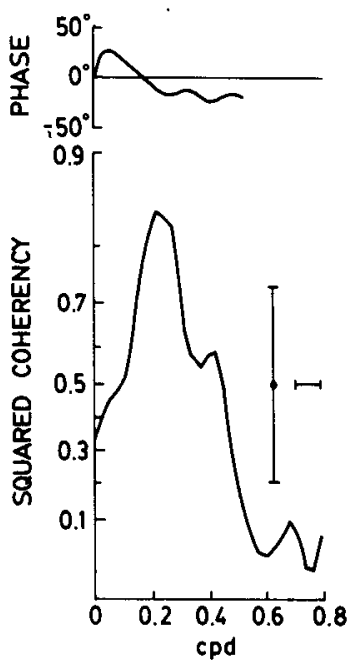

(b)

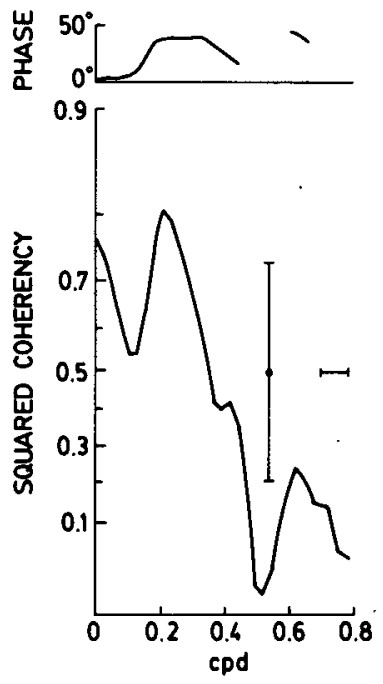

(c)
Fig. 1. (a) The experimental setup off Oregon in Cutchin and Smith (1973). The site of the current meter mooring is indicated by the square, and that of the tide gages, by the triangles. The bathymetric profile along the line extending offshore from Depoe Bay is shown as the dashed curve in Fig. 2. (b) Phase and squared coherency between $v$ from the current meter mooring and Newport adjusted sea level for the period from 5 June to 11 September 1968. Phase is positive for $v$ leading sea level and is shown only if coherency is significant. Bandwidth and $95 \%$ confidence interval are as shown. (c) Phase and squared coherency between Newport and Astoria adjusted sea level for the period from 5 June to 11 September 1968, (with Newport leading Astoria). (Redrawn from Cutchin and Smith, 1973.)

interesting and puzzling observations made during this experiment. The autospectra of sea levels, currents and atmospheric pressure are relatively smooth yet the coherence spectra all exhibit strong peaks. The observed phase differences between sea level variations along the coast do not support a simple explanation based on the resonance mechanism suggested by Buchwald and Adams (1968).

As will be discussed fully in Section 7 of this paper, we believe our resonant theory satisfactorily explains the observations of Cutchin and Smith (1973). The dominant peak at $0.22 \mathrm{cpd}$, presumably generated by some large-scale atmospheric system with this frequency, had to transfer some of its energy to the two "preferred" frequencies at around 0.40 and $0.65 \mathrm{cpd}$ due to the nonlinear terms' in the governing equations. At other frequencies, the resonance conditions were not satisfied and the transfer of energy from the main peak to these frequencies was small. The nonlinear energy transfer eventually produced a distinctively triple-peaked spectrum, as was observed.

Huyer et al. (1975) found spectral signals on the Oregon shelf which also agree with the resonance frequencies deduced from our theory. These observations are remarkable in that except for the case of surface gravity waves (e.g., see Hasselmann et al., 1973), the direct observation of nonlinear interactions in the ocean is relatively rare. Most observations of geofluid-dynamical wave-wave interactions have been made only in the laboratory.

This paper complements a number of earlier studies dealing with nonlinear effects in shelf waves. Smith (1972) and Grimshaw (1977a) have studied the modification of an individual shelf wave mode by the nonlinear terms. Grimshaw (1977b) examined sideband instability for shelf waves, while Barton (1977) looked at the generation of shelf waves by the wind via resonant interactions.

The plan for this paper is as follows: After presenting the nonlinear governing equations in Section 2, we develop the theory of resonant triad interactions for shelf waves in Section 3; in particular, we derive the equations governing the amplitudes of the individual waves in a resonant triad. Energy transfer and energy conservation are examined in Section 4, while an elementary analysis of the equations governing the wave amplitudes is given in Section 5. The theory is then specialized in Section 6 to the familiar exponential shelf profile [first introduced in Buchwald and Adams (1968)]. Our theory is finally applied in Section 7 to interpret the Oregon shelf observations. In Section 8 the problems and limitations of our theory are discussed and in Section 9 a summary and conclusion are given.

\section{Governing equations}

The nonlinear barotropic long-wave equations for a rotating system are 
$u_{t}+u u_{x}+v u_{y}-f v$

$$
=-g \eta_{x}-\frac{1}{\rho} p_{a x}+\frac{1}{\rho h} \tau_{1},
$$

$v_{t}+u v_{x}+v v_{y}+f u$

$$
\begin{array}{r}
=-g \eta_{y}-\frac{1}{\rho} p_{a y}+\frac{1}{\rho h} \tau_{2}, \\
(h u)_{x}+(h v)_{y}=-\eta_{t}-(\eta u)_{x}-(\eta v)_{y},
\end{array}
$$

where $t$ is the time, $x$ the offshore coordinate and $y$ the alongshore coordinate; $(u, v)$ are the horizontal velocity components in the $(x, y)$ direction, $\eta$ the sea surface displacement from equilibrium, $p_{a}$ the atmospheric pressure at the sea surface, $\left(\tau_{1}, \tau_{2}\right)$ the surface wind-stress components (modeled as a body force) and $h$ the depth of the ocean; $f$ and $g$ are the Coriolis parameter and the gravitational constant, respectively. We shall assume that the density $\rho$ and $f$ are constants, and that the ocean depth $h$ is a function of $x$ only.

Next, we nondimensionalize the variables $\{x, y, h$, $\left.t, u, v, \eta, \tau_{1}, \tau_{2}\right\}$ with respect to the scales $\left\{L, L, H_{0}\right.$, $\left.f^{-1}, V, V, \eta_{0}, \tau_{0}, \tau_{0}\right\}$. Assuming that the terms $f v$ and $g \eta_{x}$ in Eq. (2.1) are of comparable magnitudes, we let

$$
\eta_{0}=f V L / g \text {. }
$$

In addition, we introduce two nondimensional parameters

$$
\epsilon=V / L f, \quad \mu^{2}=L^{2} f^{2} / g H_{0},
$$

where $\epsilon$ is the Rossby number and $\mu^{2}$ the divergence parameter.

Expressing the vorticity equation [i.e., $\partial_{y}(2.1)$ $\left.-\partial_{x}(2.2)\right]$ and Eq. (2.3) in nondimensional form yields

$$
\begin{array}{r}
\left(u_{y}-v_{x}\right)_{t}+\epsilon\left[\left(u u_{x}+v u_{y}\right)_{y}-\left(u v_{x}+v v_{y}\right)_{x}\right] \\
-\left(v_{y}+u_{x}\right)=F, \\
(h u)_{x}+(h v)_{y}=-\mu^{2}\left\{\eta_{t}+\epsilon\left[(\eta u)_{x}+(\eta v)_{y}\right]\right\},
\end{array}
$$

where $F$, the wind-stress forcing term, is given by

$$
F=\frac{\tau_{0}}{\rho H_{0} f V} \frac{1}{h}\left[\frac{L}{L_{\tau}}\left(\tau_{1 y}-\tau_{2 x}\right)+\frac{h_{x}}{h} \tau_{2}\right],
$$

with $L_{\tau}$ an appropriate length scale for the wind stress.

We choose $L$ to be the width of the continental shelf/slope region $(L \approx 100 \mathrm{~km})$ and $H_{0}$ the shelf depth scale $\left(H_{0}=200 \mathrm{~m}\right)$. For shelf waves, $V$ can be taken to be $10^{-1} \mathrm{~m} \mathrm{~s}^{-1}$, and at midlatitudes $f$ is typically $10^{-4} \mathrm{rad} \mathrm{s}^{-1}$. With these choices, our nondimensional parameters have the following values: $\epsilon=0.01$ and $\mu^{2}=0.05$. Taking the wind-stress scale $\tau_{0}$ to be $10^{-1} \mathrm{~N} \mathrm{~m}^{-2}$, we have $\tau_{0} /\left(\rho H_{0} f V\right)=0.05$. Adams and Buchwald (1969) and Gill and Schumann (1974) have studied the generation of shelf waves by the wind stress. Here, however, we shall neglect the forcing term $F$ and examine only the unforced nonlinear equations.

Furthermore, we make the nondivergence approximation by neglecting the terms on the right side of Eq. (2.7). This allows us to introduce a transport streamfunction $\psi$ such that

$$
h u=\psi_{y}, \quad h v=-\psi_{x} .
$$

Substituting (2.9) into the unforced version of (2.6), and writing $h_{x}$ as $h^{\prime}$, we obtain

$$
\begin{gathered}
h\left(\psi_{x x t}+\psi_{y y t}\right)+h^{\prime}\left(\psi_{y}-\psi_{x t}\right)=\epsilon\left\{\left[\frac{h^{\prime \prime}}{h}-3\left(\frac{h^{\prime}}{h}\right)^{2}\right] \psi_{x} \psi_{y}\right. \\
+\frac{3 h^{\prime}}{h} \psi_{x x} \psi_{y}-\frac{h^{\prime}}{h} \psi_{x} \psi_{x y}-\psi_{x x x} \psi_{y}+\psi_{x} \psi_{x x y} \\
\left.+2 \frac{h^{\prime}}{h} \psi_{y y} \psi_{y}-\psi_{x y y} \psi_{y}+\psi_{x} \psi_{y y y}\right\} \cdot(2.10)
\end{gathered}
$$

Looking for waves trapped near the coast, we impose the boundary conditions $\psi \rightarrow 0$ as $x \rightarrow \infty$, and $h u=0$ at $x=0$. From Eq. (2.9), the second boundary condition becomes $\psi_{y}=0$ at $x=0$.

Next, we assume $\psi$ can be expanded as

$$
\psi=\psi^{(0)}+\epsilon \psi^{(1)}+\epsilon^{2} \psi^{(2)}+\cdots,
$$

where the $\psi^{(j) ' s}$ are independent of $\epsilon$. We also assume $\psi=\psi(x, y, t, Y, T)$ where $Y$ and $T$ are the "slow" variables defined by

$$
Y=\epsilon y, \quad T=\epsilon t .
$$

Thus, in Eq. (2.10),

$$
\frac{\partial}{\partial y} \rightarrow \frac{\partial}{\partial y}+\epsilon \frac{\partial}{\partial Y} \text { and } \frac{\partial}{\partial t} \rightarrow \frac{\partial}{\partial t}+\epsilon \frac{\partial}{\partial T} .
$$

Substituting (2.11) into (2.10), we obtain the $O(1)$ and $\mathrm{O}(\epsilon)$ equations:

$$
\mathrm{O}(1): h\left[\psi_{x x t}^{(0)}+\psi_{y y t}^{(0)}\right]+h^{\prime}\left[\psi_{y}^{(0)}-\psi_{x t}^{(0)}\right]=0,
$$

with boundary conditions $\psi^{(0)} \rightarrow 0$ as $x \rightarrow \infty, \psi_{y}^{(0)}$ $=0$ at $x=0$; and

$$
\begin{aligned}
\mathrm{O}(\epsilon): & h\left[\psi_{x x t}^{(1)}+\psi_{y y t}^{(1)}\right]+h^{\prime}\left[\psi_{y}^{(1)}-\psi_{x t}^{(1)}\right] \\
=- & h\left[\psi_{x x T}^{(0)}+\psi_{y y T}^{(0)}+2 \psi_{y Y t}^{(0)}\right]+h^{\prime}\left[\psi_{x T}^{(0)}-\psi_{Y}^{(0)}\right] \\
+ & {\left[\frac{h^{\prime \prime}}{h}-3\left(\frac{h^{\prime}}{h}\right)^{2}\right] \psi_{x}^{(0)} \psi_{y}^{(0)}+3 \frac{h^{\prime}}{h} \psi_{x x}^{(0)} \psi_{y}^{(0)} } \\
& -\frac{h^{\prime}}{h} \psi_{x}^{(0)} \psi_{x y}^{(0)}-\psi_{x x x}^{(0)} \psi_{y}^{(0)}+\psi_{x}^{(0)} \psi_{x x y}^{(0)} \\
& +2 \frac{h^{\prime}}{h} \psi_{y y}^{(0)} \psi_{y}^{(0)}-\psi_{x y y}^{(0)} \psi_{y}^{(0)}+\psi_{x}^{(0)} \psi_{y y y}^{(0)},
\end{aligned}
$$

with boundary conditions $\psi^{(1)} \rightarrow 0$ as $x \rightarrow \infty$, and $\psi_{Y}^{(0)}+\psi_{y}^{(1)}=0$ at $x=0$. 
For a shelf wave traveling parallel to a coast, we let $\psi^{(0)}$ have the form

$$
\psi^{(0)}=A(Y, T) \phi(x) e^{i(k y-\omega t)}+\text { c.c. },
$$

where c.c. denotes the complex conjugate of the preceding term. Substituting Eq. (2.15) into Eq. (2.13), and letting $c=\omega / k$, we obtain the following differential equation for $\phi$ :

$$
\begin{gathered}
-\left(-\frac{1}{h} \phi^{\prime}\right)^{\prime}+\frac{-k^{2}}{h} \phi-\frac{1}{c}\left(\frac{h^{\prime}}{h^{2}}\right) \phi=0, \\
0 \leqslant x<\infty,
\end{gathered}
$$

which, for a given value of $k$, turns out to be of the Sturm-Liouville form

$$
-\left[p(x) \phi^{\prime}\right]^{\prime}+{ }^{\prime} q(x) \phi-\lambda r(x) \phi=0
$$

as given in Boyce and DiPrima (1969, p. 493). Hence, the $O(1)$ equation reduces to a Sturm-Liouville problem with $c^{-1}$ as the eigenvalue and with boundary conditions $\phi \rightarrow 0$ as $x \rightarrow \infty$, and $\phi=0$ at $x=0$. Eq. (2.16) with an exponential shelf profile has been solved by Buchwald and Adams (1968) for the eigenvalues $1 / c^{(n)}$ and eigenfunctions $\phi^{(n)}$ corresponding to different shelf wave modes $(n=1,2, \ldots)$. By varying $k$, the dispersion curves $\omega^{(n)}(k)=k c^{(n)}$ for the different modes were also obtained.

\section{Theory of resonant interactions between shelf waves}

In this section, we will show that the presence of the nonlinear terms in Eq. (2.14) [i.e., the $O(\epsilon)$ equation] allows resonant interactions to occur between three shelf waves. We start with three distinct shelf waves of frequencies $\omega_{j}$ and wavenumbers $k_{j}(j=1,2,3)$, i.e.,

$$
\psi^{(0)}=\sum_{j=1}^{3} A_{j}(Y, T) \phi_{j}(x)
$$

$$
\times \exp \left[i\left(k_{j} y-\omega_{j} t\right)\right]+\text { c.c. }
$$

Each of the $\phi_{j}$ 's satisfies an equation corresponding to (2.16):

$$
\begin{gathered}
-\left(-\frac{1}{h} \phi_{j}{ }^{\prime}\right)^{\prime}+\frac{-k_{j}{ }^{2}}{h} \phi_{j}-\frac{1}{c_{j}}\left(\frac{h^{\prime}}{h^{2}}\right) \phi_{j}=0, \\
0 \leqslant x<\infty,
\end{gathered}
$$

where $c_{j}=\omega_{j} / k_{j}$ is the phase speed of the $j$ th wave. The boundary conditions are

$$
\phi_{j}=0 \text { at } x=0, \quad \phi_{j} \rightarrow 0 \text { as } x \rightarrow \infty .
$$

We note that the three waves described in Eqs. (3.1)-(3.3) do not necessarily correspond to three distinct shelf wave modes. Hence the superscript $n$ pertaining to the mode number has been dropped. As will be seen below (Section 7), two of the three waves in a resonant triad for the Oregon shelf are first-mode shelf waves at two different wavenumbers $\left(k_{1}\right.$ and $\left.k_{3}\right)$.

Substituting (3.1) into (2.14), and utilizing (3.2), we obtain

$$
\begin{aligned}
h\left[\psi_{x x t}^{(1)}+\psi_{y y t}^{(1)}\right]+h^{\prime}\left[\psi_{y}^{(1)}-\psi_{x t}^{(1)}\right] \\
=\sum_{j=1}^{3}\left[-\frac{h^{\prime}}{c_{j}} \phi_{j} A_{j T}-\left(h^{\prime}+2 h k_{j} \omega_{j}\right) \phi_{j} A_{j Y}\right] \exp \left[i\left(k_{j} y-\omega_{j} t\right)\right]+\sum_{l=1}^{3} \sum_{m=1}^{3}\left\{-2 \frac{h^{\prime}}{h} k_{l}^{2} \phi_{l} \phi_{m}\right. \\
\left.\quad+\left[\frac{h^{\prime \prime}}{h}-3\left(\frac{h^{\prime}}{h}\right)^{2}+k_{l}^{2}-k_{m}^{2}\right] \phi_{l}^{\prime} \phi_{m}+3 \frac{h^{\prime}}{h} \phi_{l}^{\prime \prime} \phi_{m}-\frac{h^{\prime}}{h} \phi_{l}^{\prime} \phi_{m}^{\prime}+\phi_{l}^{\prime} \phi_{m}^{\prime \prime}-\phi_{l}^{\prime \prime \prime} \phi_{m}\right\} \\
\quad \times\left(i k_{m} A_{l} A_{m} \exp \left\{i\left[\left(k_{l}+k_{m}\right) y-\left(\omega_{l}+\omega_{m}\right) t\right]\right\}-i k_{m} A_{l} A_{m}^{*} \exp \left\{i\left[\left(k_{l}-k_{m}\right) y-\left(\omega_{l}-\omega_{m}\right) t\right]\right\}\right)+\text { c.c. }
\end{aligned}
$$

In order to match the frequencies and wavenumbers on the right side of Eq. (3.4), $\psi^{(1)}$ must also be of the form

$$
\begin{aligned}
\psi^{(1)}=\sum_{\alpha} A_{\alpha}^{(1)}(Y, T) & \phi_{\alpha}^{(1)}(x) \\
& \times \exp \left[i\left(k_{\alpha} y-\omega_{\alpha} t\right)\right]+\text { c.c. }
\end{aligned}
$$

where the set of values for $\left(k_{\alpha}, \omega_{\alpha}\right)$ is given by

$$
\begin{aligned}
& \left\{\left(k_{\alpha}, \omega_{\alpha}\right)\right\}=\left\{\left(k_{j}, \omega_{j}\right) \mid j=1,2,3\right\} \\
& \cup\left\{\left(k_{l}, \omega_{l}\right) \pm\left(k_{m}, \omega_{m}\right) \mid l=1,2,3, m=1,2,3\right\} .
\end{aligned}
$$

Furthermore, we establish the following convention for indexing: for $\alpha=1,2,3,\left(k_{\alpha}, \omega_{\alpha}\right) \equiv\left(k_{j}, \omega_{j}\right)$.

We now examine the situation for $\alpha=j=1,2,3$. Substituting Eq. (3.5) into the left side of (3.4), we find that for each $j, \phi_{j}^{(1)}$ must satisfy an inhomogeneous equation

$$
\begin{gathered}
-\left(-\frac{1}{h} \phi_{j}^{(1)^{\prime}}\right)^{\prime}+\frac{-k_{j}^{2}}{h} \phi_{j}^{(1)}-\frac{1}{c_{j}}\left(\frac{h^{\prime}}{h^{2}}\right) \phi_{j}^{(1)}=f_{j}, \\
0 \leqslant x<\infty,
\end{gathered}
$$

where $f_{j}$ denotes the forcing at frequency $\omega_{j}$ and wavenumber $k_{j}$ from the right side of (3.4). [In contrast, we recall that $\phi_{j}$ satisfies the corresponding homogeneous equation (3.2) which, together with boundary conditions (3.3), constitute a standard Sturm-Liouville problem.] In nonresonant cases only the linear terms on the right side of (3.4) can contribute to $f_{j}$ 's $(j=1,2,3)$ : 


$$
f_{j}=\frac{\left[\frac{h^{\prime}}{c_{j}} \phi_{j} A_{j T}+\left(h^{\prime}+2 h k_{j} \omega_{j}\right) \phi_{j} A_{j Y}\right]}{\left[i \omega_{j} h^{2} A_{j}^{(1)}\right]} .
$$

However, if any of the following resonance conditions are satisfied, viz.,

$$
\pm\left(\omega_{1}, k_{1}\right) \pm\left(\omega_{2}, k_{2}\right) \pm\left(\omega_{3}, k_{3}\right)=(0,0),
$$

some of the nonlinear terms on the right side of (3.4) are also of frequencies $\omega_{j}$ and wavenumbers $k_{j}(j=1,2,3)$, and hence can contribute to the forcing terms $f_{j}$ 's. With no loss of generality, we write the resonance conditions as

$$
\omega_{1}+\omega_{2}+\omega_{3}=0, k_{1}+k_{2}+k_{3}=0,
$$

where $\left\{\omega_{j}\right\}$ and $\left\{k_{j}\right\}$ can have either positive or negative values.

The Fredholm alternate theorem from SturmLiouville theory (see, e.g., Boyce and DiPrima,

$$
\begin{aligned}
K_{j l m}=\int_{0}^{\infty} d x \frac{1}{h^{2}} k_{m} \phi_{j}\left\{-2 \frac{h^{\prime}}{h} k_{l}^{2} \phi_{l} \phi_{m}+\left[\frac{h^{\prime \prime}}{h}-3\left(\frac{h^{\prime}}{h}\right)^{2}\right.\right. & \left.+k_{l}^{2}-k_{m}^{2}\right] \phi_{l}^{\prime} \phi_{m} \\
& \left.+3 \frac{h^{\prime}}{h} \phi_{l}^{\prime \prime} \phi_{m}-\frac{h^{\prime}}{h} \phi_{l}^{\prime} \phi_{m}^{\prime}+\phi_{l}^{\prime} \phi_{m}^{\prime \prime}-\phi_{l}^{\prime \prime \prime} \phi_{m}\right\} .
\end{aligned}
$$

With repeated integration by parts and the help of the boundary conditions (3.3), $K_{j l m}$ can be re-expressed in another form

$$
\begin{aligned}
K_{j l m}=\int_{0}^{\infty} d x \frac{1}{h^{2}} k_{m}[ & -3 \frac{h^{\prime}}{h} \phi_{j}^{\prime} \phi_{l}^{\prime} \phi_{m}+2 \phi_{j}^{\prime} \phi_{l}^{\prime \prime} \phi_{m} \\
+\phi_{j}^{\prime \prime} \phi_{l}^{\prime} \phi_{m}- & 2 \frac{h^{\prime}}{h} k_{l}^{2} \phi_{j} \phi_{l} \phi_{m} \\
& \left.+\left(k_{l}^{2}-k_{m}^{2}\right) \phi_{j} \phi_{l}^{\prime} \phi_{m}\right]
\end{aligned}
$$

Without loss of generality, we can normalize $\left\{\phi_{j}\right\}$ so that

$$
\int_{0}^{\infty} \frac{h^{\prime}}{h^{2}} \phi_{j}^{2} d x=1
$$

Eq. (3.12) then gives us the amplitude equation for the $j$ th wave:

$$
\frac{\partial}{\partial T} A_{j}+c_{g_{j}} \frac{\partial}{\partial Y} A_{j}=-i K_{j} A_{l}^{*} A_{m}^{*}
$$

where

$$
\boldsymbol{K}_{j} \equiv c_{j}\left(\boldsymbol{K}_{j l m}+\boldsymbol{K}_{j m l}\right)
$$

and $c_{g_{j}}$ is the group velocity of the $j$ th wave (see Appendix for justification), with

$$
c_{g_{j}}=c_{j}\left(1+2 \gamma_{j} c_{j} k_{j}^{2}\right),
$$

1969 , p. 506) states that the inhomogeneous problem has a solution $\phi_{j}^{(1)}$ only if the forcing term $f_{j}$ is orthogonal to the homogeneous solution $\phi_{j}$. That is,

$$
\int_{0}^{\infty} f_{j} \phi_{j} d x=0
$$

When the resonance conditions (3.10) are satisfied, the Fredholm condition (3.11) yields

$$
\begin{aligned}
& A_{j T} \int_{0}^{\infty} \frac{h^{\prime}}{h^{2}} \phi_{j}{ }^{2} d x \\
& +A_{j Y}\left[c_{j} \int_{0}^{\infty} \frac{h^{\prime}}{h^{2}} \phi_{j}{ }^{2} d x+2 c_{j}{ }^{2} k_{j}{ }^{2} \int_{0}^{\infty} \frac{1}{h} \phi_{j}{ }^{2} d x\right] \\
& \quad=-i c_{j}\left(K_{j l m}+K_{j m l}\right) A_{l}^{*} A_{m}^{*},
\end{aligned}
$$

where $(j, l, m)$ are any of the cyclic permutations of $(1,2,3)$, and the terms on the right-hand side of (3.12) arise from the nonlinear terms in (3.4), with

$$
\gamma_{j} \equiv \int_{0}^{\infty} \frac{1}{h} \phi_{j}{ }^{2} d x .
$$

The amplitude equation (3.16) shows that, at resonance, the nonlinear terms provide a coupling mechanism between the amplitudes of the three waves. If the resonance conditions are not satisfied, the nonlinear terms do not contribute to the forcing terms $f_{j}(j=1,2,3)$, and the Fredholm condition (3.11) simply yields

$$
\frac{\partial}{\partial T} A_{j}+c_{g_{j}} \frac{\partial}{\partial Y} A_{j}=0,
$$

where each wave amplitude (at lowest order) is independent of the others, and propagate along at its own group velocity. Thus, the nonlinear terms, despite their presence only in the $O(\epsilon)$ equation, can nevertheless directly affect the $O(1)$ amplitude equations when the resonance conditions are fulfilled. How energy is exchanged between the three waves in a resonant interaction is our next topic.

\section{Conservation and transfer of energy}

In this section, we will show that the total energy of the triad is conserved even though energy is transferred between the waves.

The nondivergence or "rigid-lid" approximation employed in Section 2 allows us to consider only 
the kinetic energies of the waves, as their potential energies are very small by comparison. To lowest order, the non-dimensionalized kinetic energy per unit volume is given by

$$
\mathrm{KE} / \text { volume }=1 / 2\left(u^{2}+v^{2}\right),
$$

where the density $\rho \equiv 1$, and we have neglected the small contribution from the vertical velocity component. Next, we integrate Eq. (4.1) with the vertical coordinate $z$ running from $-h$ to 0 , the alongshore coordinate $y$ from 0 to 1 , and the offshore coordinate $x$ from 0 to $\infty$. Assuming $u$ and $v$ to be depth independent, and invoking Eqs. (2.9), the resulting kinetic energy per unit length alongshore can be expressed (to lowest order) as follows:

KE/length

$$
=\frac{1}{2} \int_{0}^{\infty} d x \int_{0}^{1} d y \frac{1}{h}\left[\left(\psi_{x}^{(0)}\right)^{2}+\left(\psi_{y}^{(0)}\right)^{2}\right] .
$$

We first consider the case of a single wave, where $\psi^{(0)}$ is given by Eq. (2.15) and then substituted into Eq. (4.2). During the integration of $y$ from 0 to 1 , $A=A(Y, T)$ is essentially constant. With $A$ written as $|A| e^{i \theta}$, we finally obtain

$$
\begin{aligned}
& \text { KE/length }=|A|^{2}\left\{\frac{1}{k}\left[\int_{0}^{\infty} d x \frac{1}{h} \phi^{\prime 2}\right]\right. \\
& \times\left.[k y-\omega t+\theta+1 / 2 \sin 2(k y-\omega t+\theta)]\right|_{y=0} ^{1} \\
& +k\left[\int_{0}^{\infty} d x \frac{1}{h} \phi^{2}\right][k y-\omega t+\theta \\
& \left.-1 / 2 \sin 2(k y-\omega t+\theta)]\left.\right|_{y=0} ^{1}\right\}
\end{aligned}
$$

Since we are interested in the variation of energy with respect to the slow time $T$, the "sine" terms representing "high" frequency oscillations can be either averaged out to zero, or simply ignored for our purposes. Thus, we have on the average

$$
\text { KE/length }=|A|^{2} \int_{0}^{\infty} d x \frac{1}{h}\left(\phi^{\prime 2}+k^{2} \phi^{2}\right) .
$$

Integrating the first term by parts, then invoking (2.16) and the normalization condition (3.15), we finally obtain

$$
\mathrm{KE} / \text { length }=-\frac{1}{c}|A|^{2},
$$

where we remind the reader that the phase speed $c$ is always negative for shelf waves.

For the triad case, we substitute Eq. (3.1) into (4.2), and perform a similar calculation. With the oscillatory terms ignored, we obtain for a triad

$$
\mathrm{KE} / \text { length }=\sum_{j=1}^{3}-\frac{1}{c_{j}}\left|A_{j}\right|^{2},
$$

which is simply the sum of the kinetic energies of the individual waves.

If we now multiply the amplitude equation (3.16) by $-A_{j}{ }^{*} / c_{j}$, we have

$$
\begin{aligned}
-\frac{1}{c_{j}} A_{j}^{*} \frac{\partial A_{j}}{\partial T}+c_{g_{j}}\left(-\frac{1}{c_{j}}\right) A_{j}^{*} & \frac{\partial A_{j}}{\partial Y} \\
& =\frac{i K_{j}}{c_{j}} A_{j}^{*} A_{l}^{*} A_{m}^{*} .
\end{aligned}
$$

Adding (4.7) to its own complex conjugate, we arrive at the energy equation for the $j$ th wave:

$$
\begin{aligned}
\frac{\partial}{\partial T}\left(-\frac{1}{c_{j}}\left|A_{j}\right|^{2}\right) & +c_{g_{j}} \frac{\partial}{\partial Y}\left(-\frac{1}{c_{j}}\left|A_{j}\right|^{2}\right) \\
& =2 \operatorname{Re}\left\{i A_{1} A_{2} A_{3}\right\}\left(-\frac{K_{j}}{c_{j}}\right),
\end{aligned}
$$

where $\operatorname{Re}\{\cdots\}$ denotes the real part of the terms inside the braces. We see that if the nonlinear coupling term on the right-hand side is not present, the energy of the $j$ th wave would simply propagate along at its group velocity $c_{g_{j}}$.

To consider the total energy of the triad, we need to sum the three separate energy equations, i.e.,

$$
\begin{aligned}
\sum_{j=1}^{3}\left(\frac{\partial}{\partial T}+c_{g_{j}}\right. & \left.\frac{\partial}{\partial Y}\right)\left(-\frac{1}{c_{j}}\left|A_{j}\right|^{2}\right) \\
& =2 \operatorname{Re}\left(i A_{1} A_{2} A_{3}\right) \sum_{j=1}^{3}\left(\frac{-K_{j}}{c_{j}}\right) .
\end{aligned}
$$

The conservation of the total energy is then expressed by the following theorem.

\section{THEOREM:}

$$
\sum_{j=1}^{3}\left(\frac{\partial}{\partial T}+c_{g_{j}} \frac{\partial}{\partial Y}\right)\left(-\frac{1}{c_{j}}\left|A_{j}\right|^{2}\right)=0
$$

Proof: Eq. (4.10) follows from Eq. (4.9) if

$$
\sum_{j=1}^{3}\left(\frac{-K_{j}}{c_{j}}\right)=0 .
$$

From Eq. (3.17), this amounts to proving

$$
\begin{aligned}
\sum_{K} \equiv K_{123}+ & K_{132}+K_{231} \\
& +K_{213}+K_{312}+K_{321}=0 .
\end{aligned}
$$

With repeated integration by parts, and the help of Eqs. (3.3) and (3.10), $K_{j l m}$ in (3.14) can be reexpressed as

$$
K_{j l m}=K_{j l m}^{(1)}+K_{j l m}^{(2)}+K_{j l m}^{(3)}+K_{j l m}^{(4)},
$$


where

$$
\begin{aligned}
& K_{j l m}^{(1)} \equiv \int_{0}^{\infty} d x \frac{1}{h^{2}}\left(1 / 2 k_{m} \phi_{j}^{\prime} \phi_{l}^{\prime \prime} \phi_{m}\right.\left.-1 / 2 k_{m} \phi_{j}^{\prime \prime} \phi_{l}^{\prime} \phi_{m}\right), \\
& K_{j l m}^{(2)} \equiv \int_{0}^{\infty} d x \frac{1}{h^{2}}\left(-3 / 2 k_{m} \phi_{j}^{\prime} \phi_{l}^{\prime} \phi_{m}^{\prime}\right)=K_{l j m}^{(2)}, \\
& K_{j l m}^{(3)} \equiv \int_{0}^{\infty} d x \frac{1}{h^{2}}\left(-k_{m} k_{l}^{2} \phi_{j}^{\prime} \phi_{l} \phi_{m}\right. \\
&\left.\quad+k_{j} k_{l}^{2} \phi_{j} \phi_{l} \phi_{m}^{\prime}\right), \\
& K_{j l m}^{(4)} \equiv \int_{0}^{\infty} d x \frac{1}{h^{2}}\left(k_{l}^{3} \phi_{j} \phi_{l} \phi_{m}^{\prime}-k_{m}{ }^{3} \phi_{j} \phi_{l}^{\prime} \phi_{m}\right) .
\end{aligned}
$$

We note that

$$
\begin{gathered}
K_{j l m}^{(1)}+K_{l j m}^{(1)}=0, \quad K_{j l m}^{(3)}+K_{m l j}^{(3)}=0 \\
K_{j l m}^{(4)}+K_{j m l}^{(4)}=0
\end{gathered}
$$

and

$$
\begin{aligned}
\sum_{m=1}^{3} K_{j l m}^{(2)}=\left(k_{1}\right. & \left.+k_{2}+k_{3}\right) \\
& \times \int_{0}^{\infty} d x \frac{1}{h^{2}}\left(-3 / 2 \phi_{1}^{\prime} \phi_{2}^{\prime} \phi_{3}^{\prime}\right)=0
\end{aligned}
$$

by the resonance conditions (3.10).

Therefore, $\sum_{K}$ can be written as

$$
\begin{aligned}
\sum_{K}= & \sum_{m=1}^{3}\left(K_{j l m}^{(1)}+K_{l j m}^{(1)}\right)+\sum_{m=1}^{3} K_{j l m}^{(2)} \\
+\sum_{m=1}^{3} K_{l j m}^{(2)}+ & \sum_{l=1}^{3}\left(K_{j l m}^{(3)}+K_{m l j}^{(3)}\right) \\
& +\sum_{j=1}^{3}\left(K_{j l m}^{(4)}+K_{j m l}^{(4)}\right)=0
\end{aligned}
$$

and our theorem for the conservation of the total energy in a resonant triad is proved.

\section{Analysis of the amplitude equations}

In recent years, the inverse scattering method has been used to solve coupled partial differential equations similar in form to our amplitude equations (3.16). (Sometimes the equations are first converted to a standard form by scaling the three $A_{j}$ 's so that the coupling coefficients $i K_{j}$ 's have the values \pm 1 .) A recent review is given by Kaup et al. (1979). Here, we shall not attempt to solve our amplitude equations in their full glory. Instead, we shall only study temporal behavior by (eventually) neglecting the spatial derivative $\partial / \partial Y$ in our equations.

If we write

$$
A_{j}(Y, T) \equiv a_{j}(Y, T) \exp \left[i \theta_{j}(Y, T)\right]
$$

where $a_{j}$ and $\theta_{j}$ are real functions, and substitute this form into our amplitude equations (3.16), the real and the imaginary parts of the resulting equations give

$$
\begin{aligned}
\frac{\partial a_{j}}{\partial T}+c_{g_{j}} \frac{\partial a_{j}}{\partial Y} & =-K_{j} a_{l} a_{m} \sin \theta, \\
a_{j}\left(\frac{\partial \theta_{j}}{\partial T}+c_{g_{j}} \frac{\partial \theta_{j}}{\partial Y}\right) & =-K_{j} a_{l} a_{m} \cos \theta,
\end{aligned}
$$

respectively, where $\theta \equiv \theta_{1}+\theta_{2}+\theta_{3}$.

The energy equation (4.8) can also be expressed as

$$
\begin{aligned}
\frac{\partial}{\partial T}\left(-\frac{1}{c_{j}} a_{j}^{2}\right)+c_{g_{j}} \frac{\partial}{\partial Y}( & \left.-\frac{1}{c_{j}} a_{j}^{2}\right) \\
& =2 a_{1} a_{2} a_{3} \sin \theta \frac{K_{j}}{c_{j}} .
\end{aligned}
$$

Focussing only on the temporal behavior, we set $\partial / \partial Y$ to zero. Eqs. (5.2) and (5.3) reduce to

$$
\begin{aligned}
\frac{d a_{j}}{d T} & =-K_{j} a_{l} a_{m} \sin \theta, \\
a_{j} \frac{d \theta_{j}}{d T} & =-K_{j} a_{l} a_{m} \cos \theta .
\end{aligned}
$$

There are many special cases one can consider. For instance, when $\theta=0$ or $\pi$, the solutions are $a_{j}$ $=$ constant, and $\theta_{j}$ varies linearly with $T$. That this situation corresponds to zero energy exchange can also be seen from the energy equation (5.4), where the energy transfer term on the right side vanishes when $\theta=0$ or $\pi$.

The case of greatest interest occurs when $\theta=1 / 2 \pi$ or $3 / 2 \pi$. For these values of $\theta$, the energy exchange is maximized, as can be seen from Eq. (5.4). Also, the phases $\theta_{j}$ are constant. For $\theta=1 / 2 \pi$, Eq. (5.5) becomes

$$
\frac{d a_{j}}{d T}=-K_{j} a_{l} a_{m}, \quad j=1,2,3 .
$$

Since the phase speeds for free shelf waves are always negative, Eq. (4.11) implies that the three $K_{j}$ coefficients cannot all have the same sign. Without loss of generality, we assume $K_{1}$ and $K_{2}$ to have the same sign, and $K_{3}$ to have the opposite sign.

The solutions of Eqs. (5.7) are the Jacobi elliptic functions. Without loss of generality, we can choose $T=0$ to be the instant when $a_{1}>0, a_{2}>0$ and $a_{3}=0$. With this choice, the initial conditions are $a_{1}(0) \equiv a_{10}>0, a_{2}(0) \equiv a_{20}>0$ and $a_{3}(0)=0$, and the solutions are

$$
\begin{aligned}
& a_{1}(T)=a_{10} \operatorname{dn}(\sigma T \mid M), \\
& a_{2}(T)=a_{20} \operatorname{cn}(\sigma T \mid M), \\
& a_{3}(T)=a_{20}\left(-K_{3} / K_{2}\right)^{1 / 2} \operatorname{sn}(\sigma T \mid M),
\end{aligned}
$$


where

$$
\sigma \equiv a_{10}\left(-K_{2} K_{3}\right)^{1 / 2}, \quad M \equiv K_{1} a_{20}{ }^{2} /\left(K_{2} a_{10}{ }^{2}\right),
$$

and $\mathrm{dn}, \mathrm{cn}$, sn are the Jacobi elliptic functions in the notation of Abramowitz and Stegun (1965, Chap. 16). Usually, the elliptic functions are restricted to $0 \leqslant M \leqslant 1$. For $M=1$, the elliptic functions reduce to hyperbolic functions, while for $M$ $>1$, the following transformations can be used:-

$$
\begin{aligned}
& \operatorname{dn}(\sigma T \mid M)=\operatorname{cn}\left(M^{1 / 2} \sigma T \mid M^{-1}\right) \\
& \operatorname{cn}(\sigma T \mid M)=\operatorname{dn}\left(M^{1 / 2} \sigma T \mid M^{-1}\right) \\
& \operatorname{sn}(\sigma T \mid M)=M^{-1 / 2} \operatorname{sn}\left(M^{1 / 2} \sigma T \mid M^{-1}\right)
\end{aligned}
$$

See Abramowitz and Stegun (1965, Chaps. 16 and 17) for properties of these elliptic functions.

Without loss of generality, we assume $0 \leqslant M \leqslant 1$. Then the period of energy transfer is given by the period $T_{d}$ of the elliptic function $\operatorname{dn}(\sigma T \mid M)$. From Abramowitz and Stegun (1965, Chaps. 16 and 17),

$$
T_{d}=2 K(M) / \sigma=2 K(M) a_{10}^{-1}\left(-K_{2} K_{3}\right)^{-1 / 2},
$$

where $K(M)$ is a complete elliptic integral of the first kind. The solutions (5.8)-(5.10) correspond, to the following situation: During the time interval $0<T<1 / 2 T_{d}$, the third wave extracts energy from the first two waves, while during $1 / 2 T_{d}<T<T_{d}$, the third wave transfers energy back to the first two waves until the initial conditions at $T=0$ are again reached at $T=T_{d}$.

As $M \rightarrow 1, K(M) \rightarrow \infty$, implying an infinitely long period for energy transfer. However, except for values of $M$ very close to unity, $K(M)$ is not a rapidly varying function. For example, as $M$ increases from zero to $0.99, K(M)$ increases monotonically from 1.57 to 3.70 . Using this fact and Eq. (5.15), we shall, in Section 7, obtain a crude estimate of the time scale of energy transfer for resonant shelf waves on the Oregon shelf.

\section{The theory applied to an exponential shelf}

So far, the depth $h$ has not been specified explicitly in our theory. In this section, we will use the Buchwald and Adams (1968) exponential shelf profile for $h$, i.e.,

$$
h= \begin{cases}H_{1} e^{2 b x}, & 0 \leqslant x \leqslant 1 \\ H_{2}, & 1<x<\infty\end{cases}
$$

where $b, H_{1}$ and $H_{2}$ are constant parameters, with $H_{2}=H_{1} e^{2 b}$. With this choice of $h$, Eq. (3.2) becomes

$$
\begin{cases}\phi_{j}^{\prime \prime}-2 b \phi_{j}^{\prime}-k_{j}^{2} \phi_{j}-\frac{2 b k_{j}}{\omega_{j}} \phi_{j}=0, & 0 \leqslant x \leqslant 1 \\ \phi_{j}^{\prime \prime}-k_{j}^{2} \phi_{j}=0, & 1<x<\infty .\end{cases}
$$

The solution of (6.2) is given in Buchwald and Adams. The resulting dispersion relation for the $n$th offshore mode is given by

$$
\begin{gathered}
\omega_{j}^{(n)}=-2 b k_{j} /\left[\left(\xi_{j}^{(n)}\right)^{2}+k_{j}^{2}+b^{2}\right], \\
n=1,2, \ldots
\end{gathered}
$$

where $\xi_{j}^{(n)}$ is the $n$th root of the transcendental equation

$$
\tan \xi_{j}^{(n)}=-\xi_{j}^{(n)} /\left(b+\left|k_{j}\right|\right), \quad \xi_{j}^{(n)}>0 .
$$

It is customary to order the $\xi_{j}^{(n)}$ 's as follows:

$$
\boldsymbol{\xi}_{j}^{(1)}<\boldsymbol{\xi}_{j}^{(2)}<\cdots \text {. }
$$

Under the normalization condition (3.15), $\phi_{j}$ corresponding to the $n$th mode is given by

$$
\phi_{j}^{(n)}=\left\{\begin{array}{c}
N_{j}^{(n)} \sin \xi_{j}^{(n)} x \exp [b(x-1)] \\
0 \leqslant x<1 \\
N_{j}^{(n)} \sin \xi_{j}^{(n)} \exp \left[-\left|k_{j}\right|(x-1)\right], \\
1<x<\infty
\end{array}\right.
$$

where

$$
N_{j}^{(n)}=\left[2 \xi^{(n)} H_{2} / b\left(2 \xi_{j}^{(n)}-\sin 2 \xi_{j}^{(n)}\right)\right]^{1 / 2} .
$$

For convenience, we now drop the modal superscript $n$.

Substituting Eq. (6.5) into (3.14), and performing the lengthy but straightforward algebra, one can show that the coefficients $K_{j l m}$ for the exponential shelf are

$$
\begin{aligned}
& K_{j l m}=N_{j} N_{l} N_{m} \frac{1}{H_{2}{ }^{2}} k_{m}\left\{b \left(-3 b^{2}-\xi_{j}{ }^{2}-2 \xi_{l}{ }^{2}\right.\right. \\
& \left.-3 k_{l}{ }^{2}-k_{m}{ }^{2}\right) I_{1}+\xi_{l}\left(-b^{2}-\xi_{j}^{2}+k_{l}{ }^{2}-k_{m}{ }^{2}\right) I_{2} \\
& +2 \xi_{j}\left(-b^{2}-\xi_{l}^{2}\right) I_{3}+\frac{\sin \xi_{j} \sin \xi_{l} \sin \xi_{m}}{\left|k_{j}\right|+\left|k_{l}\right|+\left|k_{m}\right|} \\
& \left.\quad \times\left[k_{l}^{2}\left(-2\left|k_{j}\right|-\left|k_{l}\right|\right)+\left|k_{l}\right|\left(k_{m}{ }^{2}-k_{j}^{2}\right)\right]\right\}
\end{aligned}
$$

where $I_{1}, I_{2}$ and $I_{3}$ are the following integrals:

$$
\begin{aligned}
& I_{1}=e^{b} \int_{0}^{1} \sin \xi_{j} x \sin \xi_{l} x \sin \xi_{m} x e^{-b x} d x, \\
& I_{2}=e^{b} \int_{0}^{1} \sin \xi_{j} x \cos \xi_{l} x \sin \xi_{m} x e^{-b x} d x \\
& I_{3}=e^{b} \int_{0}^{1} \cos \xi_{j} x \sin \xi_{l} x \sin \xi_{m} x e^{-b x} d x
\end{aligned}
$$

These integrals can be evaluated analytically, yielding

$$
\begin{aligned}
I_{1}=1 / 4 & {\left[\left(b A_{1}+b_{1} B_{1}-b_{1} C_{1}\right)\right.} \\
& -\left(b A_{2}+b_{2} B_{2}-b_{2} C_{2}\right)-\left(b A_{3}+b_{3} B_{3}-b_{3} C_{3}\right) \\
& \left.+\left(b A_{4}+b_{4} B_{4}-b_{4} C_{4}\right)\right], \quad(6.11)
\end{aligned}
$$




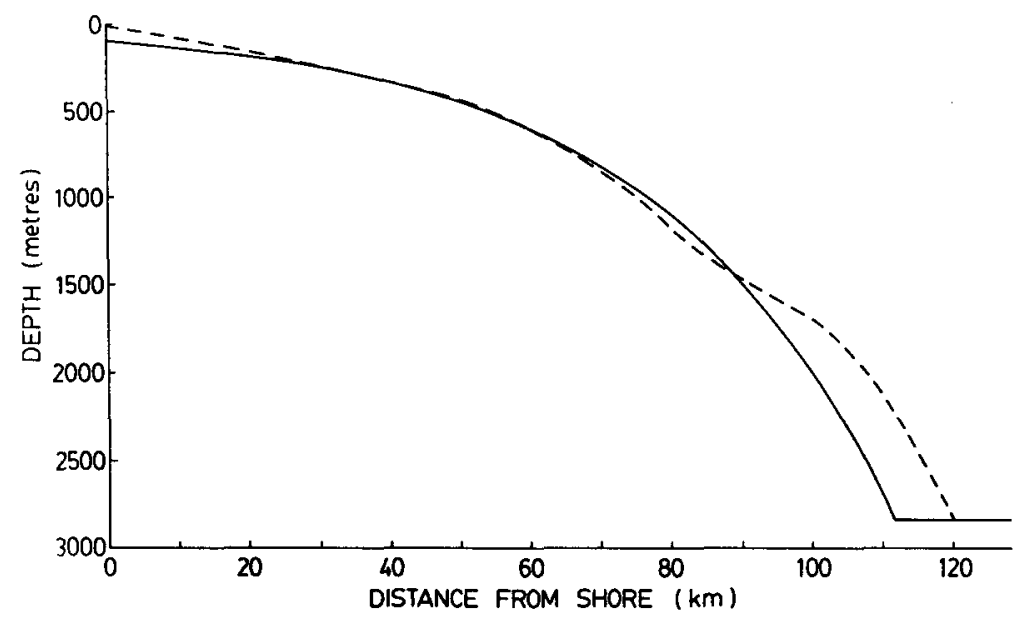

FIG. 2. The optimal fit to the topography along the line shown in Fig. 1. The dashed curve is the real topography while the solid curve is the exponential fit.

$$
\begin{aligned}
I_{2}= & 1 / 4 \\
& {\left[\left(b_{1} A_{1}-b B_{1}+b C_{1}\right)\right.} \\
& -\left(b_{2} A_{2}-b B_{2}+b C_{2}\right)+\left(b_{3} A_{3}-b B_{3}+b C_{3}\right) \\
& \left.\quad-\left(b_{4} A_{4}-b B_{4}+b C_{4}\right)\right], \quad \text { (6.12) } \\
I_{3}= & 1 / 4\left[-\left(b_{1} A_{1}-b B_{1}+b C_{1}\right)\right. \\
& +\left(b_{2} A_{2}-b B_{2}+b C_{2}\right)+\left(b_{3} A_{3}-b B_{3}+b C_{3}\right) \\
& \left.\quad-\left(b_{4} A_{4}-b B_{4}+b C_{4}\right)\right],
\end{aligned}
$$

where

$$
\left.\begin{array}{ll}
b_{1} \equiv \xi_{j}-\xi_{l}-\xi_{m}, & b_{2} \equiv \xi_{j}-\xi_{l}+\xi_{m}, \\
b_{3} \equiv \xi_{j}+\xi_{l}-\xi_{m}, & b_{4} \equiv \xi_{j}+\xi_{l}+\xi_{m},
\end{array}\right\}
$$

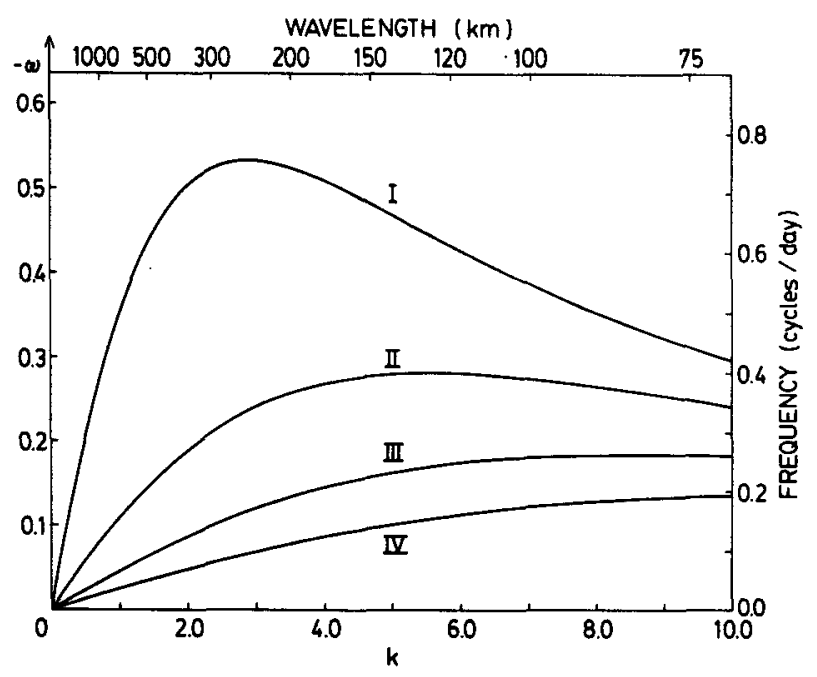

Fig. 3. Dispersion curves ( $-\omega$ versus $k$ ) for the exponential depth profile with $b=1.65$. Only the four lowest modes (I, II, III, IV) are shown. and

$$
\begin{gathered}
{\left[A_{i}, B_{i}, C_{i}\right] \equiv \frac{1}{b^{2}+b_{i}^{2}}\left[\sin b_{i}, \cos b_{i}, e^{b}\right],} \\
i=1,2,3,4
\end{gathered}
$$

\section{Applications to the Oregon shelf}

In this section, our theory is applied to the Oregon shelf and then compared with the observations in Cutchin \& Smith (1973) and Huyer et al. (1975). These two papers will henceforth be referred to as CS and HHSSP, respectively.

First, we fit the Buchwald-Adams exponential shelf profile, i.e., Eq. (6.1), to the real depth profile given in CS. With the level-off depth in deep water chosen to be $2.84 \mathrm{~km}$, the optimal fit (Fig. 2) yields the value 1.65 for the (dimensionless) parameter $b$. The variable $x$ is nondimensionalized with respect to the shelf/slope width $L(L=112 \mathrm{~km}$ from our fit), and the depth $h$, with respect to $H_{0}$, the depth scale of the shelf region $\left(H_{0}=200 \mathrm{~m}\right)$. By solving Eqs. (6.3) and (6.4) numerically, we obtain the different mode dispersion curves for the exponential profile. In Fig. 3 the curves corresponding to the first four modes are shown. (Note that we have actually plotted $-\omega$ versus $k$, since shelf waves have negative phase speeds.) Our dispersion curves for the idealized topography resemble closely the original curves in CS which have been derived using real topography.

As mentioned earlier, CS found three peaks at around $0.22,0.40$ and $0.65 \mathrm{cpd}$ in their spectral analysis of data collected on the Oregon shelf during the summer of 1968 (see Fig. 1). They also concluded from phase information that the dominant peak at $0.22 \mathrm{cpd}$ is consistent with a first-mode shelf wave with wavelength $\lambda_{1} \approx 1620 \mathrm{~km}$. 


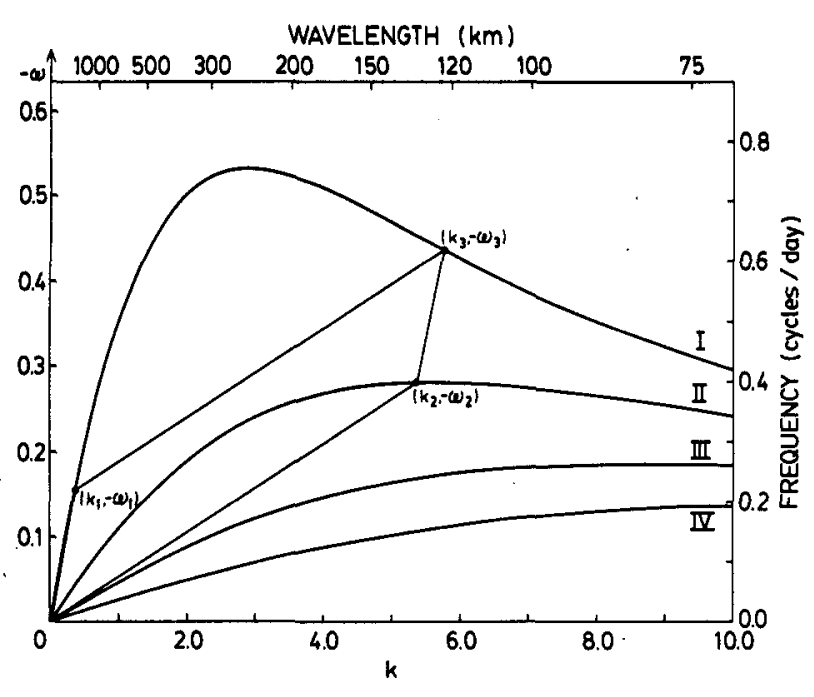

Fig. 4. The resonant triad involving waves of the lowest possible modes. With $\left(k_{1},-\omega_{1}\right)$ fixed from the observation of CS, $\left(k_{2},-\omega_{2}\right)$ and $\left(k_{3},-\omega_{3}\right)$ are then uniquely determined by constructing the parallelogram such that, with two vertices fixed at $\left(k_{1},-\omega_{1}\right)$ and $(0,0)$, the remaining two vertices touch the dispersion curves for modes I and II.

Can our resonant interaction theory explain the presence of these observed peaks? To facilitate comparison, we rewrite the resonance conditions as

$$
\omega_{1}+\omega_{2}=\omega_{3}, \quad k_{1}+k_{2}=k_{3}
$$

(which can be converted back to the original forms (3.10) by simply changing the signs of $\omega_{3}$ and $k_{3}$ ). Eqs. (7.1) can also be expressed as a vector relation

$$
\left(k_{1},-\omega_{1}\right)+\left(k_{2},-\omega_{2}\right)=\left(k_{3},-\omega_{3}\right) \text {. }
$$

If we now choose $-\omega_{1}$ to correspond to the 0.22 cpd frequency, then the dispersion curves in Fig. 3 and the vector relation (7.2) allow us to deduce the other members of the triad, namely $\left(k_{2},-\omega_{2}\right)$ and $\left(k_{3},-\omega_{3}\right)$, by a simple geometric construction (Fig. 4). The vector addition in Eq. (7.2) implies that $\left(k_{1},-\omega_{1}\right),\left(k_{2},-\omega_{2}\right),\left(k_{3},-\omega_{3}\right)$ are the three vertices of a parallelogram lying in the $(k,-\omega)$ plane, with the three vertices touching the dispersion curves, and the fourth vertex fixed at the origin. Since CS concluded that the peak at $0.22 \mathrm{cpd}$ is consistent with a first-mode shelf wave, this essentially fixes our $\left(k_{1},-\omega_{1}\right)$. Looking for interactions involving waves of the lowest possible modes, we find that $\left(k_{2},-\omega_{2}\right)$ belong to the second mode while $\left(k_{3},-\omega_{3}\right)$ belong to the first mode. With these choices for the modes, the resulting parallelogram of Fig. 4 is unique.

The resonant triad obtained from Fig. 4 is $\left(k_{1},-\omega_{1}\right)$ $=(0.382,0.155),\left(k_{2},-\omega_{2}\right)=(5.362,0.281),\left(k_{3}\right.$, $\left.-\omega_{3}\right)=(5.745,0.436)$, where these pairs are nondimensionalized with respect to the scales $\left(L^{-1}, f\right)$. The corresponding wavelengths and frequencies for the triad are $\left(\lambda_{1}, \nu_{1}\right)=(1840 \mathrm{~km}, 0.22 \mathrm{cpd}),\left(\lambda_{2}, \nu_{2}\right)$
$=(131 \mathrm{~km}, 0.40 \mathrm{cpd}),\left(\lambda_{3}, \nu_{3}\right)=(122 \mathrm{~km}, 0.62 \mathrm{cpd})$. Alternatively, we can construct our parallelogram from the original dispersion curves of CS (derived using real topography), resulting in the following values for the triad: $(1710 \mathrm{~km}, 0.22 \mathrm{cpd}),(138 \mathrm{~km}$, $0.40 \mathrm{cpd}),(127 \mathrm{~km}, 0.62 \mathrm{cpd})$. With either construction, we find excellent agreement with the observed values in CS: $\lambda_{1}=1620 \mathrm{~km}, \nu_{1}=0.22$ cpd, $\nu_{2}=0.40 \mathrm{cpd}, \nu_{3}=0.65 \mathrm{cpd}$.

There is insufficient information in CS to give a reliable estimate for $\lambda_{2}$ and $\lambda_{3}$. The phases associated with the second and third peaks in Fig. 1c are $\sim 22^{\circ}$ and $43^{\circ}$. However, the true phase can be the observed phase $+n 360^{\circ}$ (where $n$ is an integer). With the two stations $180 \mathrm{~km}$ apart, taking $n=0$ yields $\lambda_{2}=3000 \mathrm{~km}$ and $\lambda_{3}=1500 \mathrm{~km}$. But if $n$ $=+1$, then $\lambda_{2}=170 \mathrm{~km}$ and $\lambda_{3}=160 \mathrm{~km}$, which agree very roughly with the theoretical, values (131 km and $122 \mathrm{~km}$ ).

CS attempted to explain the presence of the three peaks by a mechanism proposed in Buchwald and Adams (1968). For dispersion curves which have zero slope at certain frequencies, the resulting zero group velocity implies that energy cannot propagate away, and therefore, according to Buchwald and Adams (1968), peaks in the spectrum might be expected at these frequencies. From the original dispersion curves of CS, we find that the frequencies of zero group velocity occur at $0.29,0.40$ and 0.71 cpd for modes III, II and I, respectively. The agreement between these frequencies and the observed frequencies $(0.22,0.40,0.65 \mathrm{cpd})$ appears weaker than that between our resonant triad frequencies $(0.22,0.40,0.62 \mathrm{cpd})$ and the observations. Furthermore, with the Buchwald-Adams mechanism, one has to identify the dominant peak at 0.22 cpd with a third mode wave of wavelength $<300 \mathrm{~km}$ (see Fig. 3). But, CS determined the wavelength associated with the dominant peak to be $1620 \mathrm{~km}$, which is consistent only with a first mode wave. Hence, our resonant triad mechanism seems more capable of explaining the triple-peaked spec-, trum in CS.

Next, we derive the numerical values of the coupling coefficients $K_{j}$ for the resonant triad interaction in CS. Returning to Eq. (3.10) as our resonance conditions, the signs of $k_{3}$ and $\omega_{3}$ must be reversed. Substituting in the triad values 0.382 ,

TABLE 1. The coupling coefficients (in nondimensional units) for the resonant triad interaction on the Oregon shelf re-

\begin{tabular}{|c|c|c|c|c|}
\hline$j l m$ & $K_{j l m}$ & $K_{j m l}$ & $K_{j}$ & $K_{j} / c_{j}$ \\
\hline $\begin{array}{lll}1 & 2 & 3 \\
2 & 3 & 1 \\
3 & 1 & 2\end{array}$ & $\begin{array}{c}60.6 \\
1.48 \\
-52.7\end{array}$ & $\begin{array}{c}-39.0 \\
37.7 \\
-8.02\end{array}$ & $\begin{array}{r}-8.75 \\
-2.05 \\
4.61\end{array}$ & $\begin{array}{r}21.6 \\
39.2 \\
-60.7\end{array}$ \\
\hline
\end{tabular}
ported in CS. 
$5.362,-5.745$ for $k_{1}, k_{2}$ and $k_{3}$, and $-0.155,-0.281$, 0.436 for $\omega_{1}, \omega_{2}$ and $\omega_{3}$, Eqs. (6.3), (6.6), (6.7), (6.11)(6.15), and (3.17) give the values of the coupling coefficients, as listed in Table 1.

In Section 5, we found that, with $\partial / \partial Y$ neglected, and for the case of maximum energy exchange, the solutions to the amplitude equations were given by elliptic functions. The period $T_{d}$ of energy transfer was given by Eq. (5.15). We expect $a_{10}$ to be $\leqslant 1$. (This is because the first wave dominates, hence its velocity should be comparable in magnitude to the measured velocity scale $V$, and thus with nondimensionalization, $a_{10} \approx 1$.) As $a_{20}{ }^{2}$ is significantly less than $a_{10}{ }^{2}$, we expect $M$ to be <1 from Eq. (5.11). The elliptic integral $K(M)$ increases slowly from 1.57 to 3.70 as $M$ increases from 0 to 0.99 . Taking a typical value of 3 for $K(M), 1$ for $a_{10}$, and using values of $K_{2}$ and $K_{3}$ from Table 1, we have $T_{d}$ $\approx 2.0$. Remembering $T=\epsilon t$, where $\epsilon$ is the Rossby number $(\epsilon=0.01)$ and $t$ is nondimensionalized with respect to $1 / f\left(f=10^{-4} \mathrm{~s}^{-1}\right), T_{d}$ is converted back to dimensional units on division by $\epsilon f$, yielding $T_{d} \approx 2.0 \times 10^{6} \mathrm{~s}$ or 23 days. Hence, for the triad in $\mathrm{CS}$, the time scale for energy transfer $\left(-1 / 2 T_{d}\right)$ is of order 12 days.

Huyer et al. (1975) (abbreviated HHSSP) computed rotary spectra with data collected from July to September 1972, on the Oregon and Washington shelves. Near the shore, a shelf wave should produce high coherency in the clockwise component of a rotary spectrum. In Fig. 9 of HHSSP, the clockwise portion (or, in the notation of HHSSP, the negative frequency part) of the coherency squared spectra between the Depoe Bay sea level and the $60 \mathrm{~m}$ current at the mooring $\mathrm{NH}-10$ shows four distinct signals at $0.15,0.27,0.42$ and $0.55 \mathrm{cpd}$. If one again performs the "parallelogram" construction on the dispersion curves in CS, one finds that upon associating the dominant $0.15 \mathrm{cpd}$ peak with a first-mode shelf wave, the remaining members of the triad involving the lowest possible modes turn out to be $\left(\lambda_{2}, \nu_{2}\right)=(103 \mathrm{~km}, 0.40 \mathrm{cpd})$, (second mode), and $\left(\lambda_{3}, \nu_{3}\right)=(99 \mathrm{~km}, 0.55 \mathrm{cpd})$, (first mode). Thus, it is plausible that of the four clockwise signals observed $(0.15,0.27,0.42,0.55 \mathrm{cpd})$, the last two arise from the resonant transfer of energy from the dominant peak at $0.15 \mathrm{cpd}$. The cross spectra between the current at $\mathrm{NH}-10$ and the wind at Newport (Fig. 9 of HHSSP) shows high correlation at the 0.15 and $0.27 \mathrm{cpd}$ (clockwise) frequencies, suggesting that these signals may be wind generated.

So far, we have only examined resonances involving the lowest possible shelf wave modes. Resonances involving higher modes should not be overlooked. In particular, the first three signals in $\operatorname{HHSSP}(0.15,0.27$ and $0.42 \mathrm{cpd})$, which also happen to satisfy the resonance condition (1.1), may be a resonant triad involving higher modes.

\section{Discussion}

Our theory of resonant triad interactions seems capable of explaining some of the subinertial spectral peaks observed on the Oregon shelf. Nevertheless, one must remember the many limitations of our theory: no stratification, no bottom friction or irregularities, no alongshore variations in the shelf width and no atmospheric forcing.

While CS reported barotropic motion in their measurements, HHSSP found significant baroclinic components in some of their signals, indicating stratification may be important. Furthermore, the phase speed of $356 \mathrm{~km}$ day $^{-1}$ for the $0.22 \mathrm{cpd}$ signal in CS is in excellent agreement with the theoretical phase speed of a lowest mode barotropic shelf wave (derived from their dispersion curve), while in contrast, the phase speeds in HHSSP seem substantially higher than the theoretical phase speeds for barotropic shelf waves. Stratification is known to modify the dispersion curves resulting in higher phase speeds (see Mysak, 1980, Section 3).

Bottom friction and irregularities in bottom topography (see Allen, 1980, Section 4), have also been neglected in our theory, but could be important factors. In particular, for the first-mode wave on the Oregon shelf, Brink and Allen (1978) obtained a decay time scale ( $e$-folding time) of 5 days due to bottom friction. In contrast, the time scale for resonant energy transfer is about 12 days from our theory. The actual balance between frictional effects and nonlinear energy transfer requires further investigation. Moreover, we note that the triad theory predicts two very short shelf waves (with wavelengths $\approx 100-140 \mathrm{~km}$ ), and it is conceivable that such short waves may be strongly affected or dissipated by the presence of bottom irregularities and friction.

In this paper, we have focused primarily on resonant triads involving waves of the lowest possible modes (two first-mode waves, one secondmode wave). Resonant triads involving higher modes are also possible. Bottom friction and irregularities may decide which of these triads will be excited more readily than others. Furthermore, the two secondary members of the triad extracting energy from the dominant member may in turn interact resonantly with other waves, forming additional resonant triads, and this process may be repeated.

The alongshore variation in the shelf width has also been overlooked. The changes in the width and shape of the shelf alter the dispersion curves. The triad may have formed some distance to the south where the shelf and the dispersion curves can be very different. By the time the triad reaches the region under investigation, the waves may no longer satisfy the "parallelogram" relations for a resonant triad. 
Atmospheric forcing has also been omitted in our theory. However, we must emphasize the fact that when applying our theory to the Oregon shelf, we have assumed that the dominant member of the triad is generated by (and receiving energy from) the atmospheric system. Without this input of energy, all the waves will eventually be damped out by the frictional effects. The works of Adams and Buchwald (1969) and Gill and Schumann (1974) have dealt with the generation of shelf waves by the wind. However, in addition to genuine shelf waves, Gill and Schumann (1974) found that the wind may generate forced waves which need not lie on the shelf wave dispersion curves.

Finally, we note that our theory is for interactions between three shelf waves of discrete frequencies and wavenumbers. An alternative approach is to study shelf wave interactions that occur continuously over a broad band in $\omega-k$ space (e.g., see Hasselmann, 1968). The continuum approach in general yields smaller growth rates than the discrete approach. Since distinct peaks are actually present in the spectra of CS and HHSSP, our discrete approach is probably adequate.

\section{Summary and conclusion}

Neglecting stratification, bottom friction, alongshore variations in bottom topography and atmospheric forcing, we show that the nonlinear longwave equations allow resonant interactions to occur between three shelf waves. The equations governing the amplitude and the energy of the individual waves in a resonant triad are derived. The energy equation shows that energy is transferred between the waves by the nonlinear terms, but with the total energy conserved. Neglecting the alongshore derivative $\partial / \partial Y$ in the amplitude equations, the wave amplitudes are given by elliptic functions in the case of maximum energy exchange. The theory is then applied to the familiar exponential shelf profile, where the coupling coefficients are obtained analytically.

The theory is tested against observations made on the Oregon shelf by other researchers. In their spectral analysis, Cutchin and Smith (1973) found a dominant signal at $0.22 \mathrm{cpd}$, and two secondary ones at 0.40 and $0.65 \mathrm{cpd}$. For interactions involving waves of lowest possible modes, we find that a 0.22 cpd first-mode shelf wave can, in theory, interact resonantly with (and transfer energy to) two short shelf waves of frequencies 0.40 and $0.62 \mathrm{cpd}-$ in excellent agreement with the observed frequencies. Similarly, from the rotary spectra of Huyer et al. (1975), two secondary signals observed at the clockwise frequencies of 0.42 and $0.55 \mathrm{cpd}$ also agree well with the theoretical values of 0.40 and $0.55 \mathrm{cpd}$. The time scale for energy transfer is estimated to be of order 12 days on the Oregon shelf.

We envisage the following scenario on the continental shelf: From the works of Adams and Buchwald (1969) and Gill and Schumann (1974), we believe the wind to be the dominant mechanism in generating shelf waves. However, the nonlinearity of the media allows energy to be transferred from the wind-generated spectral peak to other frequencies. Though the energy transfer is usually small, it becomes significant for certain specific frequencies and wavenumbers which happen to form a resonant triad with the dominant windgenerated signal. Secondary spectral peaks will then emerge at these resonant frequencies.

There are two notable features in our study. First, the energy transfer time scale of 12 days is relatively small when compared to the typical shelf wave periods of a few days. This is even more remarkable when one notes that the Rossby number $\epsilon$, which characterizes the size of the nonlinear terms, is only $\sim 0.01$.

Second, in the resonant interaction, the lowfrequency, long shelf wave tends to generate two higher frequency shelf waves with much shorter wavelengths, $100-140 \mathrm{~km}$. This implies a transfer of energy to the smaller scales.

The detection of these short shelf waves would provide strong support for our theory. In the meantime, we would like to point to another observation on the Oregon shelf reported in Kundu and Allen (1976), where it was found that the offshore velocity component $u$ had much shorter correlation scales in all directions than the alongshore component $v$, and that there was lower correlation between $u$ and the alongshore wind stress $\tau_{2}$ than between $v$ and $\tau_{2}$. For long shelf waves $|u| \ll|v|$, but for the short shelf waves $|u| \approx|v|$. Hence the observed $v$ would be dominated by the windgenerated long-shelf wave, but the observed $u$ would be complicated by the two short shelf waves which are also uncorrelated with the wind. Hence the presence of short shelf waves is consistent with the observations in Kundu and Allen (1976). Clearly, more observational evidence is needed to fully confirm our theory.

Acknowledgments. This work was supported by the Natural Sciences and Engineering Research Council of Canada through a graduate scholarship to WWH and Operating and Strategic Grants to LAM, for which the authors are grateful.

\section{APPENDIX}

\section{The Group Velocity}

In Section 3, we claimed that the $c_{g_{j}}$ which shows up in the amplitude equation (3.16) and defined by 
Eqs. (3.18) and (3.19) is indeed the group velocity of the $j$ th wave, i.e.,

$$
\frac{\partial \omega_{j}}{\partial k_{j}}=c_{g_{j}}
$$

\section{The proof is as follows:}

First multiply Eq. (3.2) by $\phi_{j}$, replace $c_{j}$ by $\omega_{j} / k_{j}$, and then differentiate the entire equation with respect to $k_{j}$. From this, subtract off Eq. (3.2) multiplied by $2 \partial \phi_{j} / \partial k_{j}$, yielding

$$
\begin{array}{r}
\phi_{j} \frac{\partial}{\partial k_{j}}\left(\frac{1}{h} \phi_{j}^{\prime}\right)^{\prime}-\left(\frac{1}{h} \phi_{j}^{\prime}\right)^{\prime} \frac{\partial \phi_{j}}{\partial k_{j}}-2 k_{j} \frac{1}{h} \phi_{j}^{2} \\
-\frac{1}{\omega_{j}} \frac{h^{\prime}}{h^{2}} \phi_{j}{ }^{2}+\frac{k_{j}}{\omega_{j}^{2}} \frac{\partial \omega_{j}}{\partial k_{j}} \frac{h^{\prime}}{h^{2}} \phi_{j}{ }^{2}=0 .
\end{array}
$$

Next, we integrate this equation with respect to $x$ from 0 to $\infty$. Integrating by parts and applying the boundary conditions (3.3), the first two terms cancel, leaving

$$
\begin{aligned}
& \frac{\partial \omega_{j}}{\partial k_{j}} \int_{0}^{\infty} \frac{h^{\prime}}{h^{2}} \phi_{j}{ }^{2} d x \\
& \quad=\frac{\omega_{j}}{k_{j}} \int_{0}^{\infty} \frac{h^{\prime}}{h^{2}} \phi_{j}{ }^{2} d x+2 \omega_{j}{ }^{2} \int_{0}^{\infty} \frac{1}{h} \phi_{j}{ }^{2} d x .
\end{aligned}
$$

Under the normalization condition (3.15), this reduces to Eq. (A1) with $c_{g_{j}}$ defined by Eqs. (3.18) and (3.19).

\section{REFERENCES}

Abramowitz, M., and I. A. Stegun, Ed., 1965: Handbook of Mathematical Functions. Dover, 1046 pp.

Adams, J. K., and V. T. Buchwald, 1969: The generation of continental shelf waves. J. Fluid Mech., 35, 815-826.

Allen, J. S., 1980: Models of wind-driven currents on the continental shelf. Annual Review of Fluid Mechanics, Vol. 12, Annual Reviews, Inc., 389-433.

Barton, N. G., 1977: Resonant interactions of shelf waves with wind-generated effects. Geophys. Astrophys. Fluid Dyn., 9, 101-114.
Boyce, W. E., and R. C. DiPrima, 1969: Elementary Differential Equations and Boundary Value Problems, 2nd ed. Wiley, $533 \mathrm{pp}$.

Brink, K. H., and J. S. Allen, 1978: On the effect of bottom friction on barotropic motion over the continental shelf. J. Phys. Oceanogr., 8, 919-922.

Buchwald, V. T., and J. K. Adams, 1968: The propagation of continental shelf waves. Proc. Roy. Soc. London, A305, 235-250.

Cutchin, D. L., and R. L. Smith, 1973: Continental shelf waves: Low-frequency variations in sea level and currents over the Oregon continental shelf. J. Phys. Oceanogr., 3, 73-82.

Gill, A. E., and E. H. Schumann, 1974: The generation of long shelf waves by the wind. J. Phys. Oceanogr., 4, 83-90.

Grimshaw, R., 1977a: Nonlinear aspects of long shelf waves. Geophys. Astrophys. Fluid Dyn., 8, 3-16.

- 1977b: The stability of continental shelf waves. I. Side band instability and long wave resonance. J. Aust. Math. Soc., B20, 13-30.

Hasselmann, K., 1968: Weak interaction theory of ocean waves. Basic Developments in Fluid Dynamics, Vol. 2, M. Holt, Ed. Academic Press, 117-182.

— T. P. Barnett, E. Bouws, H. Carlson, D. E. Cartwright, K. Enke, J. A. Ewing, H. Gienapp, D. E. Hasselmann, P. Kruseman, A. Meerburg, P. Müller, D. J. Olbers, K. Richter, W. Sell and H. Walden, 1973: Measurements of wind-wave growth and swell decay during the joint North Sea wave project (JONSWAP). Disch. Hydrogr. Z., A8(Suppl.), 95 pp.

Huyer, A., B. M. Hickey, J. D. Smith, R. L. Smith and R. D. Pillsbury, 1975: Alongshore coherence at low frequency in currents observed over the continental shelf off Oregon and Washington. J. Geophys. Res., 80, 3495-3505.

Kaup, D. J., A. Reiman and A. Bers, 1979: Space-time evolution of nonlinear three-wave interactions. I. Interaction in a homogeneous medium. Rev. Mod. Phys., 51, 275-309.

Kundu, P. K., and J. S. Allen, 1976: Some three-dimensional characteristics of low-frequency current fluctuations near the Oregon coast. J. Phys. Oceanogr., 6, 181-199.

LeBlond, P. H. and L. A. Mysak, 1978: Waves in the Ocean. Elsevier, 602 pp.

Mysak, L. A., 1980: Recent advances in shelf wave dynamics. Rev. Geophys. Space Phys, 18, 211-241.

Phillips, O. M., 1977: The Dynamics of the Upper Ocean. 2nd ed. Cambridge University Press, $336 \mathrm{pp}$.

Smith, R., 1972: Nonlinear Kelvin and continental shelf waves. J. Fluid Mech., 52, 379-391. 